ÉGYPTE monde arabe

\section{Égypte/Monde arabe}

14 | 1993

Dits et écrits, mémoires et rites

\title{
Penser, dire, interdire. Logiques et enjeux de la censure des écrits en Égypte
}

Analyse de presse

\section{Bernard Botiveau}

\section{(2) OpenEdition}

\section{Journals}

Édition électronique

URL : https://journals.openedition.org/ema/579

DOI : $10.4000 /$ ema. 579

ISSN : 2090-7273

Éditeur

CEDEJ - Centre d'études et de documentation économiques juridiques et sociales

Édition imprimée

Date de publication : 30 juin 1993

Pagination : 133-162

ISSN : 1110-5097

Référence électronique

Bernard Botiveau, « Penser, dire, interdire. Logiques et enjeux de la censure des écrits en Égypte», Égypte/Monde arabe [En ligne], 14 | 1993, mis en ligne le 08 juillet 2008, consulté le 07 juillet 2022. URL : http://journals.openedition.org/ema/579; DOI : https://doi.org/10.4000/ema.579

Ce document a été généré automatiquement le 7 juillet 2022

Tous droits réservés 


\section{Penser, dire, interdire. Logiques et enjeux de la censure des écrits en Égypte}

Analyse de presse

\section{Bernard Botiveau}

«Ainsi Dieu propose en paraboles le vrai et le

faux. L'écume s'en va au rebut, mais ce qui est utile aux hommes reste sur la terre. Dieu propose ainsi des paraboles.»

Coran, sourate al-ra'd, XIII, 17, cité par Farag

Fawda $^{1}$

1 La délégation d'oulémas qui, le 7 janvier 1992, lors de la $24^{\mathrm{e}}$ Foire du livre du Caire, est parvenue à faire retirer de l'exposition et de la vente une dizaine d'ouvrages d'auteurs égyptiens, a donné le signal d'une année 1992 qui, pour nombre d'écrivains et d'intellectuels, restera dans les annales de l'édition égyptienne comme une «année noire ». Le débat est relancé sur une pratique dont personne ne feint de croire qu'elle peut être instantanément mise à bas, mais dont chacun est conscient qu'il est urgent d'en discuter les contours et de lui inventer de nouvelles limites. Un nombre important d'intellectuels se sont mobilisés à partir des faits dont il est question et ont débattu de leur fonction dans la société, compte tenu des limites qui leur paraissent à présent tracées par des instances de décision dont ils ont du mal à comprendre sinon les mobiles, du moins les stratégies et à définir des réponses appropriées.

2 Les écrivains d'aujourd'hui peuvent identifier ce que fut la "longue marche » de leurs ânés tour à tour pris aux pièges de la censure anglaise, de la censure monarchique ou de celle de l'État nassérien. Ils ont appris, en lisant les mémoires de leurs historiens, à anticiper sur l'interdiction pour mieux en déjouer les pièges et ils ont observé, dans l'expectative, l'ouverture sadatienne, souvent vite refermée. A présent, s'il existe toujours des logiques de l'interdiction intellectuelle, un consensus semble s'imposer parmi les intellectuels pour considérer que de plus en plus, ces logiques s'articuleront 
autour de nouveaux clivages, résultant de changements récents dans les allégeances politiques et de l'évolution des stratégies des gouvernants.

3 Le « mérite » des oulémas est probablement de rappeler que la logique de l'interdiction n'est pas simplement externe. Externe, elle l'est certainement lorsque, pendant la guerre du Golfe, les opposants à l'intervention égyptienne contre l'Irak ne peuvent que difficilement se faire entendre. Elle l'est encore, quoique de façon biaisée, lorsque la question de l'interdiction est posée sur la base d'un livre - les Versets sataniques perçu comme l'élément d'un « complot » de l'Occident contre les musulmans. Mais elle apparaît surtout interne lorsqu'un écrivain égyptien de faible renommée - 'Ala Hamed - devient en 1990, pour un petit nombre de ses confrères, le «nouveau Rushdie égyptien ", tandis que pour la majorité d'entre eux il n'est qu'un écrivain égyptien, sans doute pas des meilleurs, mais un écrivain comme un autre, menacé par une interdiction de parole dont les implications sont concrètement évaluées, en dépit de dénonciations parfois très générales $d u$ "pouvoir politique» ou du «pouvoir religieux». Et ce d'autant plus qu'en juillet 1992, un écrivain souvent contesté par ses pairs, Farag Fawda, est assassiné comme représentant le plus visible de ce pourquoi ces écrivains affirment sans relâche se battre : la liberté de s'exprimer avec un minimum de détours et de périphrases, y compris dans l'expression littéraire la plus stricte.

4 La complexité du problème est à la mesure de l'importance des recompositions de la scène politique égyptienne depuis plusieurs années et qui se traduisent par le brouillage des repères intellectuels, l'incertitude des alliances ou le manque de visibilité des institutions de l'État et de la société. On ne comprend donc plus guère comment fonctionne la censure, pourtant bien présente. Car elle est en effet souvent paradoxale : des livres longtemps sur le marché font soudain l'objet d'une interdiction, tandis qu'il est possible de se les procurer sans guère de difficulté au Caire ${ }^{2}$. Certains de ces ouvrages connaissent même beaucoup de succès hors de l'Égypte, grâce par exemple aux éditeurs libanais ou algériens et aux traductions en langues européennes ${ }^{3}$. Parfois, des fonctionnaires de la censure dénoncent celle qu'exerce « illégalement » une institution comme al-Azhar. Les exemples sont multiples. Il faut donc, dans un premier temps, s'interroger sur les modes de fonctionnement de l'interdiction pour mieux en cerner la réalité, telle que la perçoivent les intellectuels concernés. C'est-à-dire répondre aux questions du quoi (qu'est-ce qui fait l'objet d'un débat sur l'interdiction), $\mathrm{du}$ comment (comment se pratique l'interdiction, quelles en sont les règles reconnues et identifiées) et du qui (quels acteurs interviennent dans ce débat sur l'interdiction et cherchent à l'infléchir). S'agissant de ces acteurs, il faut en second lieu tenter de comprendre les logiques de leurs interventions et les enjeux majeurs pour lesquels ils se mobilisent.

5 Une précision terminologique s'impose : le terme le plus employé par la plupart des commentateurs pour désigner tout ce que recouvrent les différentes formes de censure de la pensée et de l'expression, est celui de musâdara, qui correspond littéralement à la confiscation, la saisie matérielle d'oeuvres littéraires et artistiques conformément à la loi, même quand il s'agit d'une loi d'exception. Dans le contexte dont il est question ici, la musâdara vise plus largement toute forme de limitation arbitraire de la pensée et de l'expression, que ce soit par la censure préalable (raqâba), la saisie judiciaire conservatoire (tahaffuz) ou la confiscation (musâdara). Le terme est le plus souvent employé, du moins par ceux qui la combattent, pour désigner la censure arbitraire, ou l'interdiction intellectuelle (musâdarat al-fikr). 
Les objets de la censure

6 Pour tout censeur, un « bon livre " semble être un livre dont l'auteur se cantonne à une fiction dont les termes sont suffisamment neutres et explicites pour apparaître détachés des questions débattues quant à l'identité culturelle et politique de l'Égypte ; ou à une étude sociologique, économique ou juridique remplissant, des fonctions d'expertise ou de pédagogie dans tel secteur de l'activité sociale. Quant à ce qui est de nature à susciter quasi-spontanément un débat polémique, il faut semble-t-il distinguer deux niveaux: conformément à une "coutume» bien établie, on ne peut sans précautions traiter de faits concernant les trois éléments centraux de la stratification identitaire de l'Égypte : égyptien, arabe et musulman. Cette tradition bien établie et souvent reconnue par les intellectuels correspond à une donnée de l'histoire à la fois ancienne et récente de l'Égypte et ne pose pas aujourd'hui les problèmes les plus cruciaux; en revanche, ce qui est beaucoup plus problématique aujourd'hui, c'est tout ce qui touche à la réécriture de cette histoire en fonction, d'une part, des débats en cours en Égypte et, d'autre part, des procédures de légitimation en vigueur pour déterminer ce qui est constitutif de l'identité de la nation égyptienne aujourd'hui.

7 En ce qui concerne le premier niveau d'intelligibilité, j'emprunterai un exemple à la production cinématographique et théâtrale. Dans un article récent, un critique de cinéma ayant par ailleurs occupé des fonctions de censure préalable au Ministère de la Culture dressait, non sans ironie, la liste de tout ce qu'il était interdit à un auteur de théâtre ou de cinéma de montrer en public, s'agissant de l'Égypte. Décrivant une circulaire de 1947, il ne relevait pas moins de 33 interdictions dans une première catégorie concernant le « domaine social et moral » et 31 dans une seconde concernant la « sécurité et l'ordre public » :

«Ainsi, il est interdit d'évoquer la puissance divine par des choses sensibles, de figurer les prophètes, de montrer la lecture du Coran dans les rues, dans tout endroit non propice ou par un récitant chaussé, de montrer un cercueil ou des femmes suivant le mort. Il est également interdit de porter atteinte à la réputation de l'Égypte et des pays frères en montrant des ruelles sales, des charrettes à âne, des voitures à bras, des vendeurs ambulants ou des étameurs (...), la mendicité et les mendiants (...).»

8 La liste s'étendait aux façons de montrer la famille égyptienne, les notables (titres, grades et décorations) et les hommes politiques, la vie intime des individus, la bienséance en matière médicale, les exécutions capitales, les suicides ou toute scène "de violence et de brutalité excessives ». Dans la seconde catégorie était visée toute scène montrant des menées communistes ou subversives, antimonarchiques ou antiparlementaires, des grèves ou autres actes de "sédition ». Et il ajoutait que bon nombre de ces interdictions pouvaient se retrouver aujourd'hui comme elles avaient existé au début de ce siècle, même si les termes et les catégories de la dénonciation avaient évolué au rythme des changements révolutionnaires et des recompositions politiques.

9 Ce type de critique se retrouve assez couramment pour la production intellectuelle actuelle, et dans la volumineuse production de commentaires sur la censure depuis la Foire du livre de 1992, des remarques éparses attestent de la permanence de ces interdits et parfois, d'un regain de virulence à leur propos. Ainsi, le film Mariage à l'égyptienne, dont l'auteur est une chercheuse égyptienne de l'Université américaine du Caire, Rîm Saad Luqa, présenté au cours de l'été 1992 au festival du film documentaire d'Isma'iliyya, a déclenché une mini tempête. Le film montre la vie d'une femme 
abandonnée par son mari et qui vit dans un quartier populaire du Caire dont sont décrites, sans complaisance, les difficiles conditions d'existence. Certains commentateurs s'en prennent au film, comme l'historien 'Abd al-'Azîm Ramadan qui estime, dans le quotidien al-Wafd, que «le film nuit à la réputation de l'Égypte $e^{6}$ ". Signe que les temps ont changé, Muhammad 'Awda réplique' :

«Rîm n'a pas inventé ce quartier, elle ne s'est pas servie de décors ni n'a écrit un scénario sur mesure pour des acteurs professionnels. Mais elle a filmé la réalité d'un quartier dans lequel vivent et souffrent une majorité de gens. «

De tels exemples peuvent être pris dans la littérature : nous aurons l'occasion d'y revenir à propos du cas de Naguib Mahfouz dont le rejet par une partie de l'opinion égyptienne ne tient pas seulement à ce qu'il laisse entendre de ses perceptions de l'islam, mais aussi à ce que ses romans donnent à voir de l'Égypte ; même si, en Égypte, le livre n'est pas - comme le cinéma et le théâtre - soumis à la censure préalable mais au régime de la saisie après publication. A côté de ces débats quasi rituels, il est possible de percevoir des modalités différentes d'intervention des acteurs et une autre organisation du discours sur ce qu'il est permis de penser et d'écrire en Égypte.

11 Un deuxième niveau d'intelligibilité s'organise en effet autour de la question de savoir comment l'Égypte, dans ses projets politiques et culturels, dans son organisation sociale et dans ses relations extérieures, peut et doit être représentée. Si, bien sûr, le traitement de l'histoire islamique dans sa relation à l'histoire égyptienne est actuellement central, comme l'indique l'interventionnisme croissant d'al-Azhar dans la vie publique, le débat s'articule aussi autour des acquis de l'histoire récente, acquis « incontestables » pour beaucoup, indépendamment de leurs affiliations idéologiques et partisanes. L'Égypte est une nation dotée d'un État moderne, tout le monde en convient, mais les clivages apparaissent au moment de définir qui a le droit, et dans quel but, de s'approprier la mémoire et les institutions de cet État-nation.

Des références à l'Umma des musulmans apparaissent de ci de là, mais leur fréquence et leurs significations "rituelles " semblent sans commune mesure avec celles faites à la patrie-nation (watan) égyptienne, œuvre et résultat d'une sédimentation politique incluant depuis le XIXe siècle l'État de Muhammad Ali et de ses successeurs, la période du Libéral age, Nasser, Sadate et à présent Moubarak. Faruq Husni, ministre de la Culture, situe la liberté de l'écrivain dans le cadre de la nation égyptienne telle qu'elle apparaît à l'époque de Moubarak ${ }^{8}$. Sous Nasser, la culture fut régie par un Ministère de l'Orientation nationale et par celui de l'Instruction publique. Elle est à présent régie par le Ministère de la Culture, dont l'une des attributions est de veiller à ce que l'écrivain tout écrivain égyptien - puisse écrire sans entraves et que ses droits d'auteurs soient respectés. Un ministère "moderne ", attentif non seulement aux conditions politiques de la création, mais aussi à ses conditions sociales et économiques ; une modernité qui, comme le dit autrement Sâmi Khachaba, nous force à «ne pas capituler (...) devant tous ceux qui prétendraient nous imposer un retour au Moyen-Age" ».

13 L'appel à une citoyenneté garante de l'égalité des Egyptiens entre eux est présent, sous des apparences fréquemment contradictoires, dans de nombreuses prises de position. C'est le cas pour le poète Ahmad 'Abd al-Mu'ti Higâzi, qui s'exprime en ces termes dans une de ses chroniques d'al-Ahrâm ${ }^{10}$ :

"Je voudrais avant tout insister sur une chose dont beaucoup de gens n'ont pas conscience, bien qu'elle aille de soi, c'est que le fait de tenir également aux valeurs religieuses et morales ne signifie pas que la protection de ces valeurs doive être 
monopolisée privativement par quelque institution ou groupe. C'est au contraire un devoir national (wâgib qawmi) que nous avons à remplir ensemble, car les valeurs religieuses et morales sont au fondement de toute société et de notre société en particulier. C'est là un premier argument. Un autre est que l'islam est une religion dont tous les croyants sont en charge ensemble, et non une classe de clercs revendiquant pour eux-mêmes le privilège de soumettre les autres à leur autorité ainsi qu'à leurs interprétations et opinions propres. »

Accolée à cette affirmation de foi républicaine, l'insistance sur les valeurs religieuses et morales trahit, mieux que bien d'autres déclarations, l'embarras dans lequel se trouvent à présent nombre d'intellectuels qui n'ignorent plus que le peuple égyptien peut, dans le contexte actuel, être perçu comme étant aussi un peuple musulman. Cela semble aller de soi pour les grands oulémas, quand ils jouent sur un amalgame entre citoyens et croyants (musulmans), à l'instar du cheikh d'al-Azhar ${ }^{11}$ :

« La créativité ne permet pas à un écrivain de dévier du bon sens, lorsqu'il parle ou écrit à propos de ce qui constitue le coeur de la croyance, d'autant que s'il fait cela, il porte également atteinte aux sentiments des citoyens et à leur foi et se moque de leur raison et de leur intelligence, alors qu'il devrait respecter et protéger les valeurs comme cela est admis dans toutes les croyances. (., :) Ceux qui, au nom de la créativité, s'opposent aux fondements de la religion et à ses dogmes établis ne font que s'opposer aux textes du Coran $\{. .$.$\} . »$

Le médiatique cheikh Cha'rawi est plus direct lorsqu'il livre son appréciation des suites judiciaires de l'affaire 'Ala Hamed, cet écrivain condamné pour ses écrits à huit ans de prison [cf. infra $]^{2}$. Il félicite le président Moubarak d'avoir su résister à l'appel de ceux " ceux qui font peu de cas de la religion" qui lui demandaient d'abroger, comme la loi le lui permet, la décision de justice prise à rencontre de l'écrivain : "Son attitude est celle d'un prince musulman qui refuse qu'on s'en prenne à l'islam, l'Umma islamique dans son ensemble le soutient dans son refus d'abroger le jugement contre un individu qui a insulté les prophètes ». Son hommage à l'État égyptien, défenseur du citoyen égyptien-musulman, est appuyé par celui qu'il adresse au juge 'Umar 'Abd al-Raziq, auteur de la condamnation à la prison de 'Ala Hamed: «Ce jugement est une décoration posée sur la poitrine des nobles magistrats égyptiens ».

16 Tout semble autoriser actuellement de telles assertions, à commencer par les appréciations portées par les plus hautes autorités de l'État. Dans sa rencontre avec une délégation d'écrivains lors de la Foire du livre de 1992, le président Moubarak a évoqué l'affaire de la saisie d'ouvrages par des oulémas et le cas de 'Ala Hamed ${ }^{13}$. Il a dit l'importance du rôle de la justice et de son "indépendance ", la liberté de l'écrivain et la nécessité de la tolérance et, en ce qui concerne l'histoire et les sciences islamiques, l'importance de l'ijtihâd. Mais il a aussi insisté sur le cadre dans lequel doivent se dérouler les débats publics : tout écrivain se doit de respecter d'une part les religions, d'autre part al-Azhar, "phare des sciences religieuses éclairées et citadelle de la pensée islamique ». Une même accentuation peut être relevée dans le cas de Faruq Husni qui, toutes précautions prises en faveur de la liberté de création, n'en précise pas moins que la liberté de l'individu ne saurait se confondre avec celle de la collectivité et que la créativité est insérée dans un système de droits et de devoirs réciproques: "Nous sommes un peuple croyant, nous avons des coutumes et des choses sacrées qui doivent être respectées ${ }^{14} . "$ tenir compte de ses citoyens non musulmans, comme le fait par ailleurs Tharwat Abàza, 
président de l'Union des écrivains, lorsqu'il stigmatise en 'Ala Hamed l'écrivain «qui choque les sentiments des musulmans et des chrétiens ${ }^{15}$ » et déclare qu'il y va de «la paix sociale, de la sécurité publique et de l'unité nationale ${ }^{16} »$. Mais c'est une idée de l'unité nationale où entre moins la diversité égyptienne que le souci de tenir compte de la réislamisation du discours politique, imposée par les mouvements de l'islam politique et traduite en termes plus diplomatiques par al-Azhar. L'exemplification du rôle d'alAzhar confirme que l'État égyptien compte sur les oulémas pour diffuser une idéologie intégratrice fondée sur un islam rationnel, mais ceux-ci sont parfois prompts à rappeler que cet équilibre ne saurait se trouver que dans une organisation assurant la prééminence de la religion musulmane ${ }^{17}$.

18 L'opposition maintes fois manifestée par les oulémas au juge 'Achmâwi tient, entre autres raisons, au fait que l'interprétation de l'islam qu'il propose est au contraire fondée sur une confiance dans les capacités intégratrices des valeurs islamiques, qui permettraient un dépassement de ces clivages «communalistes». Il est donc logique pour lui que la souveraineté de l'Égypte l'emporte sur celle de l'islam ${ }^{18}$. Moins connu, un historien azharien spécialiste de l'histoire mamelouke de l'Égypte, Ahmad Subhi Mansur, est régulièrement frappé d'interdit par al-Azhar pour proposer ce même type d'interprétation, ou le terme watan reçoit la prééminence sur celui de umma sans que la rationalité de là philosophie politique de l'islam s'en trouve selon lui affectée $e^{19}$.

19 La délimitation du cadre de référence s'organise ainsi selon une gradation allant de ceux qui intègrent la liberté de création dans un ensemble d'Egyptiens sans distinction spécifique autre que l'appartenance nationale, ceux qui privilégient simplement l'appartenance à l'une des "trois religions » et ceux qui doublent cette appartenance d'une hiérarchisation sous la direction de l'islam. Dans le premier cas, on trouvera tous ceux qui mobilisent le réfèrent du libéral age, particulièrement explicite, comme nous le verrons plus loin; avec les invocations de Taha Husayn, Abbas al-'Aqqad, Mansur Fahmi et autres Muhammad Khalaf Allah ${ }^{20}$. Dans les second et troisième cas, la frontière est ténue et rassemble en tout cas bien souvent al-Azhar et les militants de l'islam politique. Ainsi, pour le cheikh d'al-Azhar, Gadd al-Haqq Ali Gadd al-Haqq,

«la censure n'est pas une fin en soi, mais le moyen de vérifier que les écrits respectent ce qui est socialement sain, la croyance, les mœurs, les valeurs attachées au comportement. Elle vise aussi à écarter (de Ia société) tout ce qui lui nuit, de sorte que la jeunesse ne dérive pas vers des idées extérieures aux principes de l'islam et de sa charî'a $a^{21}$."

La même restriction est formulée par le journal al-Cha'b, tribune du Parti du travail et de certaines tendances islamistes, quand il rappelle que les « trois religions » ont droit à un égal respect, mais ajoute: "Tout écrivain ou penseur appartient à la religion musulmane, dont il doit respecter les interdits et les choses sacrées".» On ne peut mieux illustrer ce recentrage qu'en comparant les appréciations portées sur la GEBO (General Book Organization / Office national du livre), après que la maison d'édition gouvernementale, qui avait réédité l'ensemble des livres de Farag Fawda au lendemain de son assassinat, en juillet 1992, eut décidé de les retirer de la vente en janvier 1993 $\left(25^{\mathrm{e}} \text { Foire du livre }\right)^{23}$. Après l'interdiction de 1993, un autre auteur censuré par al-Azhar, le poète Hasan Teleb", estime que "l'on n'arrive plus à distinguer la GEBO d'a-Azhar ", tandis que l'Organisation égyptienne des droits de l'homme (OEDH) fait état d'un rapport qu'elle a adressé à Samir Sarhan, président de la GEBO, pour l'interroger sur la 
réalité « des nombreuses plaintes qu'elle a reçues d'écrivains et de romanciers dont les œuvres ont disparu des librairies alors qu'elles avaient été éditées par la GEBO elle-même ${ }^{25}$ ». Ces mises en garde de différents commentateurs, qui rappellent que la GEBO n'appartient en propre à personne, renvoient à un autre type d'interprétation quant à la "nationalité " de la maison d'édition, dont on citera comme exemple celle de Saber 'Abd al-Dayem, wakil de la Faculté de langue arabe de l'Université de Zagazig :

«Lorsque la GEBO - institution nationale que le peuple finance à la sueur de son front et au travers de ses luttes - fait imprimer plus de six (sic) ouvrages du Dr. Farag Fawda en une seule fois, et quand on sait que sa pensée choque la conscience populaire égyptienne, faite de piété instinctive et d'attachement aux principes d'un islam pur, l'encouragement d'une maison d'édition nationale pour une telle pensée constitue un véritable imprimatur en faveur de la diffusion de telles pensées parmi la jeunesse, comme si elles devaient être citées en exempte. Quant à nous, nous désirons pour la jeunesse une pensée éclairée et modérée qui approfondisse dans les esprits une vision pieuse, au lieu de cette pensée qui défigure le dogme et égare les jeunes dans des labyrinthes et des conflits intellectuels éloignés de la réalité et de l'environnement dans lesquels elle vit $^{26}$."

L'interne et l'externe

21 Ces débats autour de la liberté d'expression et de création ont des significations et des fonctions internes à l'Égypte, mais ils ne sont pas à l'abri des effets de l'environnement international. La guerre du Golfe, ce "séisme brutal ", est bien présente dans ce contexte et elle a amplifié l'effet désastreux déjà produit par l'affaire Rushdie; le fait que, dans certains médias, des intellectuels taxent d'anti-islamiques des écrits d'auteurs égyptiens s'inscrit, certes, dans les logiques internes du débat sur la réislamisation et sur l'application de la charîa à la production intellectuelle, mais il dépend aussi de la conscience qu'ont ces critiques de l'image caricaturale qu'ont fréquemment donnée de l'islam et de sa culture les médias occidentaux. La virulence avec laquelle un écrivain comme 'Ala Hamed - «ce Rushdie égyptien ${ }^{28}-$ est pris à partie par quelques intellectuels ne s'explique d'abord que parce que son cas peut être rapproché de celui de Rushdie, bien qu'il soit sans commune mesure avec lui. Rappelons que dans l'affaire Rushdie, la très grande majorité des intellectuels égyptiens avaient soit pris leur distances, soit mis l'accent sur l'inscription de cette affaire dans un vaste "complot» occidental anti-islamique". Pour "Abd al-Wudud Chalabi, l'évocation de l'affaire Hamed par la revue Newsweek aggrave le cas de cet " écrivaillon » en même temps qu'il rend suspecte toute intervention de journaux étrangers qui pourraient au moins s'apercevoir que, même en Occident, tout ne peut pas s'écrire quand il s'agit de religion, voire de la réputation des gouvernements ${ }^{30}$.

Pourtant, les deux affaires ne sont pas comparables de ce point de vue. Le danger, visiblement perçu par une forte majorité de commentateurs qui prennent la défense de 'Ala Hamed en tant que victime de la liberté de créer ${ }^{31}$, est que tous les écrivains égyptiens suspectés de dissidence par les tenants de "l'orthodoxie » religieuse soient désormais classés dans le camp des "ennemis de l'intérieur» que dénonçait notamment Fahmi Huwaydi lors de l'affaire Rushdie ${ }^{32}$. C'est là pour eux un seuil infranchissable, dont la définition caractérise cette nouvelle étape dans laquelle est entrée la critique de la licéité ou de l'illicéité en matière religieuse ; c'est une raison, pour tout intellectuel conscient de ses responsabilités, de se mobiliser. 
L'interaction permanente entre l'interne et l'externe se mesure aussi en fonction des transformations récentes qui ont eu lieu dans le monde arabe et musulman. D'une certaine manière, la pertinence des politiques de réislamisation est pour certains confirmée par les succès obtenus ailleurs par l'islam politique, de même qu'elle est combattue par d'autres pour des raisons symétriques. L'impact des politiques iranienne et soudanaise est évident de ce point de vue, comme le montre par exemple l'intervention de l'écrivain Husayn Ahmad Amin, reproduite par al-Cha'b comme un échantillon édifiant de la pensée laïciste ${ }^{33}$ :

«(...) l'islam n'a jamais rien dit de la vie politique et sociale, il ne peut que diriger la vie individuelle et spirituelle. L'État islamique est une farce qui conduit à la catastrophe en quelques années comme on l'a vu en Iran et au Soudan. »

Mais c'est surtout autour, de la situation algérienne que se sont cristallisées ces polémiques, le débat sur la censure, relancé par les événements de la Foire du livre de janvier 1992, ayant été fortement amplifié dans les semaines suivantes par le coup d'état militaire survenu à Alger le 11 janvier. Le fait que le même auteur se soit félicité de ce que l'armée algérienne ait empêché la formation d'un gouvernement islamique démontre pour al-Cha'b "l'attitude hostile à la démocratie et à la volonté du peuple de Husayn Ahmad Amin ". Il faut dire que l'écrivain a établi une comparaison sans équivoque entre l'Algérie et l'Égypte, justifiant à ses yeux l'interdiction des "partis islamiques » en Égypte. Répondant en eftet à l'analyse de Makram Muhammad Ahmad, président du syndicat des journalistes et rédacteur en chef d'al-Musawwar, pour qui « l'Égypte n'est pas l'Algérie », H. Ahmad Amin précise ${ }^{35}$ :

«L'Égypte souffre des mêmes problèmes que l'Algérie, le danger que représentent les fondamentalistes n'est pas moindre que ce qu'il est en Algérie et c'est donc à juste titre que le président Moubarak a interdit les partis religieux; et je pense même que, dans des élections libres en Égypte, les islamistes l'emporteraient. A mon sens donc, la situation en Algérie ne diffère pas de celle de l'Égypte. »

Quelle que soit la valeur de la comparaison, il n'en reste pas moins que ceux qui dénoncent "l'amalgame" peuvent être aussi ceux qui n'hésitent pas à faire un rapprochement entre les deux pays, à l'instar toujours d'al-Cha'b pour lequel le gouvernement égyptien n'a pas su dénoncer « le coup d'État-contre le projet islamique en Algérie ${ }^{36}$ ». 'Adil Hamûda prête même aux oulémas qui ont fait interdire des ouvrages à la Foire du livre l'intention d'emboîter le pas aux islamistes algériens qui ont su les premiers montrer le chemin à suivre, dont la première étape est le contrôle de la production intellectuelle $e^{37}$. Certains semblent toutefois hésiter à s'engager dans une telle comparaison, soit qu'ils ne la jugent pas pertinente, soit qu'ils craignent qu'elle n'amplifie la polémique. Il faut cependant souligner que l'évocation de l'Algérie a lieu en particulier à propos de l'évaluation du rôle joué par les oulémas d'al-Azhar que 'Achmâwi - dont L'islam politique a été édité en Algérie - et,quelques autres soupçonnent de "flirter avec les islamistes" et de profiter de l'écho donné à la situation en Algérie pour justifier leur intervention dans le débat public ${ }^{38}$.

Auteurs et ouvrages interdits

26 Bien que la question de l'application de la charî'a et de l'islamisation de la société articule l'essentiel de ces débats, elle est aussi le prétexte à différentes interrogations sur la démocratisation de l'expression intellectuelle et plus généralement de la vie politique. Des, questions telles que : «Qu'est-ce qu'être égyptien? Quelles lectures de l'histoire égyptienne et islamique sont légitimes? Quelle place faire aux courants de 
l'islam politique dans le cadre d'un véritable pluralisme?» établissent entre, les défenseurs de la liberté intellectuelle des clivages qui ne favorisent pas également dans leur défense tous les intellectuels ayant eu à subir l'interdiction et parfois la sanction. Il serait certainement arbitraire de pousser trop loin les distinctions, dans la mesure où tous ces auteurs subissent des modalités assez proches d'interdiction, mais on constate que les auteurs contestent fréquemment l'intervention d'al-Azhar, estimant que si ces ouvrages parlent de l'islam, ils n'en parlent pas de la même façon, et en tout cas parlent aussi des conditions, variables dans le temps, qui ont permis que le débat soit transporté sur le terrain de l'islamisation. Ici, l'héritage nasséro-sadatien est sollicité dans bien des cas. On peut ainsi distinguer, pour en rendre plus visibles les nuances, plusieurs catégories d'ouvrages contestés et défendus.

Parmi les écrits que nombre d'auteurs s'emploient à présenter comme ne concernant pas l'islam mais la société égyptienne et qui, par conséquent, ne devraient pas relever d'une critique «islamique», on citera les œuvres des romanciers et des poètes ${ }^{39}$. L'argument le plus souvent invoqué est qu'il s'agit là d'oeuvres littéraires et que de l'usage de la métaphore, des symboles et des mythes, on ne saurait inférer aucun manque de respect à la morale collective, ce type d'écrits témoignant de constructions de l'imaginaire où l'islam n'est pas a priori concerné. Le droit de l'écrivain à son écriture est vigoureusement défendu dans les cas de Naguib Mahfouz (relativement protégé par son prix Nobel), Edouard al-Kharrat ${ }^{40}$, Hassan Teleb et Ibrahim 'Issa'. Naguib Mahfouz, dont Les enfants de notre quartier (Awlâd hâretna] a eu droit à une méchante réplique du cheikh Kishk (« Notre avis sur Les enfants de notre quartier ») s'en explique dans un dialogue avec l'écrivain Gamal al-Ghitâni ${ }^{42}$ : al-Jablawi, le héros du roman, ainsi que les autres personnages et le quartier lui-même doivent être pris pour ce qu'ils sont et non comme représentant des figures et des lieux de la mythologie religieuse. Ailleurs, il précise son étonnement devant la critique des oulémas ${ }^{43}$ :

«L'attitude de nos savants et de nos experts en religion est difficile à comprendre. Il ne faut pas confondre art et religion, le problème essentiel étant ce qui n'apparaît pas au moment de la lecture du roman. Or le roman doit être lu comme un roman et non comme de l'histoire, et la lecture doit se faire sur un plan esthétique et non religieux. "

Le cas de 'Ala Hamed est plus complexe car la qualité littéraire de ses nombreux écrits, "dont beaucoup à compte d'auteur", ne jouit pas d'un grand crédit auprès de ses confrères, sans parler du caractère "mégalomaniaque » qu'ils lui prêtent («l'auteur se couvre d'éloges dans sa quatrième de couverture » et il aurait, en 1988, " écrit au ministre de la Culture pour demander le prix Nobel »)". L'auteur du voyage dans le cerveau d'un homme (Masâfa fí 'aqq rajûl) décrit le déplacement d'un homme dans le temps et dans la mémoire. Dans un propos outré, Ahmad Bahgat cite la description de certaines péripéties de son périple ${ }^{45}$. Au paradis, par où il commence, il croise Adam, qu'il moque pour sa faute, puis vient Moïse qui s'acharne à frapper le sol de son bâton sans parvenir à lui arracher ni eau, ni serpent, ni le moindre lézard. Quant à Noé, assis devant un bassin rempli d'eau, il confectionne un bateau de papier qu'il s'amuse à faire flotter.

Aussi différents soient-ils, ces auteurs représentent en quelque sorte l'attente d'autres écrivains qui pensent avoir conquis leur droit de s'exprimer, sous réserve qu'ils se montrent prudents dans leur façon de décrire la réalité sociale. C'est cet héritage, chèrement conquis, qu'il y a lieu de défendre. Quant aux essais de 'Adil Hamuda, Qanâbil wa masâhif [Des bombes et des exemplaires du Coran] et de Sanâ al-Masri, Wara' 
al-hijâb [Derrière le voile], il s'agit " d'ouvrages politiques et non-religieux ${ }^{46}$. Hamuda, qui explique comment Sadate a utilisé les courants religieux musulmans - al-jihâd en particulier - et chrétiens pour atteindre certains de ses objectifs intérieurs et extérieurs, a écrit un livre dans lequel, précise fRose af-Yussef. «ne figure aucune citation $d u$ Coran ni de la tradition du Prophète ". Quant à Sana al-Masri, elle analyse l'interprétation que donnent les Gamâ'ât (associations islamistes, ndlr) du statut de la femme dans la société. Si elle utilise des sources coraniques et traditionnistes, c'est pour mieux contester l'utilisation qu'en font les islamistes, à l'instar des courants « radicaux » qui se sont manifestés au cours de l'histoire. Il s'agit autrement dit d'un ijtihâd différent de celui d'al-Azhar et des Gamâ'ât, et son livre doit, pour l'hebdomadaire, être lu comme un ouvrage de sociologie et non comme un ouvrage sur l'islam et encore moins contre l'islam; par exemple, lorsqu'elle critique les arguments habituellement avancés pour justifier que la femme se consacre à ses obligations familiales, arguments liés à ses «aptitudes» ou au fait que le travail féminin serait source de chômage pour les hommes égyptiens. L'islam, ce n'est pas forcément cela.

Cet ouvrage peut sans doute être considéré comme traitant des interprétations politiques de l'islam et non de l'islam lui-même dans ses dogmes. Mais il s'apparente aussi à une autre catégorie d'ouvrages, ceux dont le dogme de l'islam est au centre du débat. De ce point de vue, Muhammad Sa'îd al-Achmâwi a concentre sur lui l'essentiel des attaques des oulémas depuis plusieurs années. La première édition de Usûl al-charî'a date de 1979 et c'est seulement en 1987, avec l'édition de L'islam politique, ouvrage fortement médiatisé, que le débat a commencé à prendre de l'ampleur. Les idées de 'Achmâwi sont connues mais il faut en rappeler brièvement le principe. Partant d'une réflexion sur l'histoire de l'islam, il emboîte le pas de 'Ali 'Abd al-Ràziq en rappelant que l'islam n'est pas une « religion politique », que rien, dans la philosophie islamique, ne permet de développer une théorie de l'organisation sociale et politique des peuples musulmans. La politique n'est pas sacrée et le choix des dirigeants ne relève en rien de la compétence des spécialistes de l'écriture que sont les clercs, mais au contraire du principe électif; du choix du peuple.

31 En ce sens, les islamistes d'aujourd'hui sont ces "nouveaux kharidjites " ${ }^{48}$ héritiers de différents mouvements sectaires qui se sont imposés par leur radicalisme dans l'histoire. Cette critique vise aussi les oulémas-juristes, «oulémas du pouvoir » dont les ouvrages visaient à légitimer les pouvoirs en place, fussent-ils des usurpateurs. Mais 'Achmâwi ne s'en tient pas aux militants politiques actuels. Il affirme qu'en dépit des apparences, les oulémas d'aujourd'hui font le lit de l'islam politique, comme le montrent leur participation aux banques islamiques, leur rejet de toute réforme du droit islamique (notamment en ce qui concerne le droit de la famille) ou leur intervention dans la vie publique en faveur de l'application de la charî'a à toute activité sociale et individuelle- De plus, al-Azhar regroupe en son sein des oulémas que 'Achmâwi considère comme explicitement islamistes. Les grands oulémas pourraient ne voir là qu'une critique supplémentaire à toutes celles qu'ils ont déjà essuyées au cours de ce siècle et qui, de toute évidence, ne vient pas du sérail. Le problème est qu'elle est émise par un magistrat égyptien connu et de haut rang, juriste professionnel connaissant suffisamment le droit islamique pour mettre en difficulté ses interprètes qualifiés et ayant un accès privilégié aux grands médias. La menace est assez précise pour justifier des rappels à l'ordre réguliers d'al-Azhar, coordonnés avec des attaques directes de juristes en position de le faire. C'est le cas de Tawfiq Chawi, membre de 
l'association des Frères musulmans, qui a introduit une plainte auprès d'al-Azhar contre les ouvrages de 'Achmâwi et exige la destitution d'un juge qui «a failli à sa mission ${ }^{49}$.

Des auteurs moins protégés peuvent être plus vigoureusement combattus, surtout s'ils sont de purs produits de l'Université islamique. Ahmad Subhi Mansur, un historien qui a soutenu en 1977, à al-Azhar, une thèse sur le soufisme dans l'Égypte mamelouke, n'a pu se présenter devant son jury de thèse que parce qu'il avait supprimé les deux tiers de son texte original ${ }^{50}$. Au cours des années suivantes, plusieurs des livres qu'il avait publiés sur l'histoire islamique ont été interdits sur intervention d'al-Azhar ${ }^{51}$ et il a même été emprisonné, "sur la base d'une accusation intellectuelle montée de toutes pièces ". Trois raisons expliquent l'ardeur mise à le combattre ; il s'agit d'un azharien, il propose un ijtihâd différent et, surtout, il conteste à la fois la compétence des grands oulémas et le bien-fondé du contrôle exercé par eux sur les écrits. A la question qui lui est posée dans une interview publiée par l'hebdomadaire al-Ahâli - «Doit-on comprendre, de ce que vous dites, que vos adversaires (les oulémas) flirtent avec le courant religieux parce qu'il sera bientôt au pouvoir ?' »-, il a cette réponse qu'il est intéressant de citer ${ }^{53}$ :

«Peut-être certains d'entre eux font-ils ainsi, mais de façon générale, je dois dire qu'ils occupent des postes et ont des pouvoirs sans avoir de compétences scientifiques ou en ijtihâd; ils n'ont pas le temps de lire et de faire de la recherche et c'est pourquoi ils considèrent le mujtahid qui innove comme un danger pour leurs postes et pour leurs fonctions. Le danger ne concerne pas l'islam, al-Azhar elle-même ou la nation, mais le fait que soient démasquées leur insuffisance scientifique et leur paresse intellectuelle, aussi se défendent-ils en interdisant la pensée et ses œuvres, ce qui s'est précisément produit dans mon cas. »

La rationalité de la censure

33 La focalisation de la critique sur al-Azhar ne doit pourtant pas masquer les modalités administratives et juridiques par lesquelles elle s'exerce, quand il ne s'agit pas de simples voies de fait. Nombre d'intellectuels stigmatisent le rôle des oulémas, qu'ils estiment usurpé compte tenu des conditions politiques de l'Égypte actuelle, et analysent le mécanisme de l'interdiction sur le plan juridique et politique. Le rôle d'alAzhar ne paraît pas moins central, mais il est intégré à un mécanisme plus complexe dont jouent les institutions, tant dans leurs relations avec les intellectuels que dans les justifications qu'elles donnent de leur propre intervention en direction de l'opinion. Il faut à présent examiner les modalités pratiques de la censure et ses justifications juridiques, ce dernier aspect étant manifestement le plus important, car c'est le plus souvent au nom de l'État de droit que les intellectuels critiquent les multiples interventions qui viennent d'être évoquées, considérant que beaucoup d'entre elles pourraient être évitées si le droit égyptien était correctement appliqué.

Nous avais déjà relevé certains paradoxes de la censure qui en rendent le fonctionnement souvent obscur. L'observation des différentes affaires survenues depuis la condamnation, en 1990, de 'Ala Hamed, confirme cette remarque. Des ouvrages ont ainsi été retirés de la vente et de l'exposition lors de la Foire du livre du Caire de 1992, ainsi que des librairies, sur simple décision d'un éditeur craignant les réactions d'al-Azhar. Dans d'autres cas, ce sont les oulémas eux-mêmes qui sont intervenus sur place, à la Foire du livre, pour faire retirer des ouvrages de la vente. Dans la plupart des cas, l'interdiction, temporaire ou définitive, résulte d'une décision administrative et/ou judiciaire : si la décision est généralement judiciaire, l'initiative 
en est prise par le parquet de telle juridiction, par un ministère ou par al-Azhar même, par exemple dans le cas qui vient d'être cité de Ahmad Subhi Mansur, contre lequel l'Université islamique a déposé une plainte auprès de la Cour supérieure de sûreté de l'État. Qu'en est-il du droit?

On relèvera tout d'abord que la Constitution égyptienne protège en principe la liberté de création, mais en la soumettant à des restrictions correspondant au statut des intellectuels défini par le nassérisme (rôle « constructif » de l'intellectuel au service de l'idéologie nationale) et aux périodes exceptionnelles (régime de l'état d'urgence, en vigueur actuellement $)^{54}$ :

Art. 47 : La liberté d'opinion est garantie. Chaque individu a le droit, dans les limites de la loi, d'exprimer et de publier sa pensée, oralement, par écrit, en images ou par tout autre moyen d'expression. L'autocritique et la critique constructive constituent une garantie pour le bien-fondé de l'édifice national.

Art 48: La liberté de la presse, de l'impression et de l'édition ansi que de tous les moyens d'information est assurée. La censure sur la presse est interdite. Sont également interdits, par voie administrative, l'avertissement, la suspension ou la suppression des journaux. Par exception, en cas de proclamation de l'état d'urgence ou en temps de guerre, une censure limitée peut être imposée à la presse, aux imprimés et aux moyens d'information, relativement à des questions d'intérêt général ou dans des buts de Sécurité nationale. Le tout en conformité avec la loi.

Ce syncrétisme juridique du régime de l'interdiction est mis en œuvre par un certain nombre de textes qu'il serait vain de citer dans leur intégralité, hors d'une étude spécifique sur cette question. Six textes importants ont jalonné cette évolution et constituent actuellement à la fois le stock des normes protectrices de la liberté de créer et l'arsenal répressif qui en est l'envers ${ }^{55}$. Le texte le plus ancien est la loi ${ }^{\circ} 20$ de 1936 sur le régime des imprimés. Il prévoit qu'en cas de conflit, la saisie d'écrits n'appartient qu'au Conseil des ministres. Le code pénal contient plusieurs articles définissant des crimes et délits commis par la voie de publication d'écrits : écrits contre «l'ordre établi » et la Constitution (art. 174), contre les cultes (art. 161 : «Le fait d'imprimer ou de publier un livre reconnu comme saint par l'un des cultes publiquement professés en altérant intentionnellement le texte de ce livre de manière à en dénaturer le sens»); des peines de prison et d'amende sont prévues dans ces différents cas (art. 98). La loi nº 345 de 1954 sur la protection des droits des auteurs inscrit le rôle de l'écrivain dans le cadre défini par le Ministère de l'Orientation nationale (actuellement Ministère de la Culture), tandis que la loi $\mathrm{n}^{\circ} 62$ de 1958 définit l'état d'urgence (hâlat al-tawârî\}. C'est en application de cette loi que sera réinstitué, le jour de l'assassinat de Sadale, l'état d'urgence (Déclaration du 6 octobre 1981). Dans ce régime d'exception, actuellement en vigueur et renouvelé régulièrement (tous les deux ans) en Égypte, toute décision concernant l'interdiction ou tout appel contre une telle décision appartient exclusivement au président de la République - en sa qualité de chef d'état-major des armées - ou à son représentant. Enfin, la loi n 103 de 1961 sur la réforme d'al-Azhar accorde à l'Académie des recherches islamiques (institut azharien de 50 membres choisis parmi les grands oulémas) un certain droit de regard sur la production des écrits : l'Académie a pour mission de "renouveler la culture islamique et de l'expurger de ses redondances et de ses scories, ainsi que des effets du fanatisme politique et confessionnel », mais aussi, de " donner son avis sur les problèmes d'école ou les problèmes sociaux qui se rapportent au dogme $»^{56}$. 
L'objectif de la loi de 1961 était d'intégrer al-Azhar à l'État égyptien et d'en faire l'un des relais de l'idéologie nassérienne en direction des Egyptiens musulmans, qu'elle contrôlait dans son système éducatif et les institutions qui dépendaient d'elles. En 1961, le texte ne pouvait être lu que d'une certaine façon ; aujourd'hui, les oulémas se savent en mesure d'en proposer une interprétation différente qui ne limite plus l'activité de l'Académie au contrôle des éditions du Coran, de ses commentaires et autres biographies du Prophète. Il leur permet d'affirmer leur compétence à émettre des avis en direction des institutions de contrôle et notamment de la magistrature, pour tout ce qui concerne les "problèmes sociaux ». C'est bien contre cela que réagissent beaucoup d'intellectuels quand ils estiment qu'al-Azhar outrepasse ses prérogatives, comme le rappelle l'éditeur de 'Achmâwi et de Sanâ al-Masri (Sînâ' li-l-nachr) dans un « droit de réponse » au quotidien al-Akhbâr". Quoiqu'il en soit, selon une opinion assez courante, seules trois institutions ont leur mot à dire dans les affaires de censure : la justice d'abord, sous le contrôle de laquelle cette affaire doit être placée en règle générale, puis le chef de l'État et le Conseil des ministres dans le cadre des lois précitées, mais perçus comme instances d'arbitrage et non de répression ${ }^{58}$. Quant à la justice, elle a précisé sa position dans un arrêt rendu par la Cour de cassation ${ }^{59}$ :

«La loi n¹03 de 1961 sur al-Azhar ne confient pas de texte autorisant al-Azhar ou l'Académie des recherches islamiques à demander la saisie conservatoire de livres. Le rôle de l'Académie se borne dans ces affaires à suivre les recherches et études qui se publient sur l'islam et le turâth islamique et à déterminer ce qui est sain dans les opinions émises et ce qui doit être amendé et appelle une réponse. »

D'autres dysfonctionnements de l'ordre juridique au détriment des créateurs sont souvent signalés, dont il faut relever les plus graves aux yeux de ces commentateurs. Tout d'abord l'affaire 'Ala Hamed où, rappelons-le, l'auteur, l'imprimeur et le diffuseur ont été condamnés à huit ans de prison et $5.000 \mathrm{LE}$ d'amende. Or, la sanction prévue par le code pénal est de 6 mois à 5 ans d'emprisonnement, et de 500 à 1.000 LE d'amende pour, les faits tendant à provoquer la sédition confessionnelle (art. 98) ou portant atteinte à l'une des trois religions publiquement reconnues (an. 161), sans qu'il soit possible de faire cumuler les peines (art. 32) ${ }^{60}$. Dans la même affaire, le jugement, prononcé par la Cour de sûreté de l'État, section de l'état d'urgence (première instance) n'a pas été confirmé, comme il aurait dû l'être en vertu de la loi sur l'état d'urgence, par le gouverneur militaire, en l'occurrence le chef de l'État ${ }^{61}$. Enfin, il est rappelé que les institutions ordinairement habilitées à opérer des saisies sont le parquet et les services de police chargés des œuvres artistiques et littéraires, sous le contrôle exclusif de la juridiction compétente ${ }^{62}$.

Ces faits relèvent sans doute de ce que les sociologues du droit considèrent comme faisant partie de «l'indétermination du droit». Dans le contexte dont il est question, surtout compte tenu des législations d'exception qui sont en cause, ils sont perçus par ceux qui s'expriment sur ces questions comme une cause d'incertitude et surtout comme un facteur d'arbitraire, alors qu'en présence de "voies de fait", la seule réponse encore envisageable est le recours à la juridiction ordinaire, dans un pays dont les instances dirigeantes mettent régulièrement en avant le principe de souveraineté de la loi. Toujours est-il que le droit constitue le lieu d'un débat sur le principe de son utilisation par la société civile et spécialement par ceux qui s'en instituent les représentants, à savoir les intellectuels. 

recherché, en ce qui concerne les écrits touchant la religion, dans la préservation des prérogatives d'un corps de spécialistes, en l'occurrence les oulémas, comme, le cheikh le reconnaît involontairement en répondant aux questions d'un journaliste sur les divergences autrefois assumées par les philosophes musulmans ${ }^{68}$ :

« la philosophie a ses lois et celui qui s'y adonne doit être un spécialiste comme dans les autres sciences; c'est pourquoi elle ne doit pas être abandonnée à la multitude. Diffusée dans le public par de pseudo-philosophes, elle n'est que tromperie et n'a rien à voir avec la religion. De même, ceux qui tirent argument de l'existence, dans le passé, de sociétés de philosophes, oublient avec quelle facilité les médias dont nous disposons aujourd'hui répandent pour le meilleur et pour le pire des informations et des idées parmi lesquelles il est difficile de séparer le bon grain de l'ivraie. Il n'y a donc aucune raison de protester d'un passé où la discussion philosophique avait lieu entre initiés et ne touchait pas tes masses. »

44 Si donc la loi est "claire " ${ }^{69}$, elle ne l'est pas pour tout le monde. Nous l'avons déjà relevé pour nombre de commentateurs opposés à la législation et surtout aux interprétations arbitraires qui en sont faites. On relèvera à cet égard les fréquentes allusions à l'iniquité de pratiques qui, certes, correspondent à des « voies de fait », mais 
qui trouvent aussi une légitimité dans des «lois scélérates ». Réformer la loi ce peut être, par un retournement du sens et pour reprendre l'analyse des Frères musulmans, supprimer purement et simplement les législations de la censure, car dans une société islamique qui ne .tolère pas d'institutions religieuses, ces législations ont perdu leur raison d'être, comme le souligne l'un des idéologues de l'association lors d'un colloque de l'OEDH sur la liberté d'opinion et de croyance ${ }^{70}$ :

«La censure qui précède la saisie est fondamentalement une fonction séculière et ne relève pas de l'obligation religieuse. Dans la conception islamique en effet, la liberté de croyance et de pensée est absolue et il n'existe pas en islam ce que l'on appelle des institutions religieuses. (...) Les essais, sous quelque forme que ce soit devraient sortir du cercle de l'interdit, ce qu'il faudrait qu'un texte énonce clairement. »

La pression des oulémas

Al-Azhar, qui observait une attitude discrète depuis la réforme que lui avait imposée le pouvoir nassérien, devient, pour la très grande majorité des intervenants, un acteur central. Une pièce d'un dispositif plus vaste, disent certains comme nous l'avons vu, où l'État égyptien, l'Université islamique et les Frères musulmans recherchent un équilibre de nature à éviter le conflit, autrement dit la fitna. Les intérêts convergent, sans doute temporairement, entre ces institutions dont les relations ont été façonnées dans le conflit au cours de plusieurs décennies, et cet équilibre nouveau, même s'il est fragile, est perçu comme une menace par les intellectuels qui craignent de faire les frais d'une «islamisation molle» destinée à éviter le pire. Le régime de la censure et de l'interdiction en est le signe.

Trois éléments permettront de préciser cette visibilité croissante de l'Université islamique en tant qu'acteur politique : son auto-représentation, les représentations de l'extérieur et son nouveau "fondamentalisme", donnée récente La façon dont le cheikh d'al-Azhar présente l'Université est double ${ }^{71}$. D'une part, l'Université islamique doit remplir sa mission traditionnelle de protection du dogme tout en la conciliant avec les impératifs du projet national défini après 1952. D'autre part, la nation égyptienne est composée dans sa majorité de citoyens musulmans qui sont menacés dans leur identité par les excès de la modernité de l'État-nation. Les oulémas ont une fonction bien précise et eux seuls sont à même de la remplir dans la mesure où ils détiennent, par leur formation et leur mode de reproduction en tant que groupe, les clés de l'interprétation de l'histoire collective. En prétendant exercer un contrôle sur la production intellectuelle en Égypte, les oulémas ne sortent donc pas de la légalité, c'est-à-dire des compétences définies par la loi de 1961.

Telle n'est pas l'analyse de certains écrivains. La revue Adab wa naqd [Littérature et critique] estime que l'institution «tente en permanence de se rapprocher du pouvoir politique » et que, en dépit de surenchères et d'oppositions périodiques, l'affaire semble se résumer "en une sorte d'accord tacite, non écrit, entre le pouvoir politique et l'autorité religieuse $^{72}$ ». Une tradition "interventionniste» dans la culture égyptienne, masquée par l'emprise nassérienne, réapparait sournoisement dans de multiples et minimes actions. «Une cuvre soumise à al-Azhar ne revient pas » (et donc n'est pas diffusée), estime un écrivain. Les éditeurs craignent l'avis d'al-Azhar, nous l'avons relevé. Un ensemble de faits qui justifie la crainte que le monopole d'un magistère religieux ne s'installe en Égypte, comme le dit Ahmad 'Abd al-Mu'ti Higâzi ${ }^{73}$, d'autant que la compétence des oulémas peut être révoquée en doute. C'est l'argument de Hassan Teleb, poète interdit 
dont les vers ont été comparés à certains passages du Coran et qui proteste de ce que la pratique de la poésie transmise en particulier de l'époque abbaside, a enseigné ce style de poésie et a été exemplifiée par de très nombreux poètes dans le passé.

Qu'al-Azhar cherche à tirer le meilleur parti de ses relations avec le gouvernement dans la période actuelle, nul n'en doute. Mais la critique la plus incisive, formulée d'abord, nous l'avons vu, par M. S. al-'Achmâwi, est certainement que l'on peut d'ores et déjà penser qu'un " accord tacite » lie aussi une partie non négligeable des oulémas aux militants actuels de l'islam politique, à commencer par les Frères musulmans. C'est aussi l'avis de l'OEDH, quand elle estime que "l'Académie des recherches islamiques de l'Azhar s'aligne sur certaines Gamâ'ât de l'islam politique qui sont sorties de la légalité " ${ }^{74}$. Les termes dans lesquels l'Académie a condamné 'Ala Hâmed sont, de fait, révélateurs de ce point de vue $\mathrm{e}^{75}:$ «L roman contient des idées favorables à l'athéisme et à l'incroyance et niant les religions révélées, et appelle à changer la société par la révolution. «

Cette critique, qui s'alimente de plusieurs constats (rapprochement des Frères musulmans et des grands oulémas, jugements positifs sur l'expérience soudanaise notamment, défense des islamistes algériens, "défense et illustration » d'un conservatisme social qui serait justifié par une "saine" lecture de la charîa est présentée de façon plus subtile et plus convaincante par ceux qui se demandent si l'on doit attribuer au hasard le fait que les livres « mis à l'index » par l'Université islamique sont bien souvent des ouvrages qui soit s'en prennent explicitement aux « extrémistes » (mutatarrifin ), soit sont justiciables - quant aux thèmes qu'ils traitent - de leurs anathèmes: Fawda ${ }^{76}$, 'Achmâwi ou autres Sanâ al-Masri. 'Adil Hamuda traduit cette question .dans les termes suivants: "Pourquoi al-Azhar, qui combat l'extrémisme, saisit-il des livres dirigés contre l'extrémisme ?" "

Nostalgie du liberal age et défense de l'héritage nassérien

50 Si la culture islamique s'impose aujourd'hui comme le cadre dans lequel se discute la validité de l'interdiction, elle n'est pour beaucoup qu'un prétexte pour réévaluer les référents historiques susceptibles de fonder des valeurs politiques et sociales nouvelles, de nature à conforter un « État de droit » en réalité fort menacé, mais présenté souvent comme la condition de la démocratisation qui se trouve ainsi au centre des débats. Il doit être indiqué ici que le libéralisme de l'entre-deux-guerres n'est pas systématiquement opposé au nassérisme et à ses prolongements, mais qu'il s'agit de deux moments constitutifs de l'identité égyptienne actuelle. Cette constatation faite, on s'étonnera donc peu que par exemple al-Ahâli, s'appuyant sur une série d'entretiens avec Naguib Mahfouz, Nabil al-Hilâli, Muhamad al-Damati et Muhammad 'Imara, écrive que «l'époque de Taha Husayn était plus éclairée que ce que nous connaissons aujourd'hui ".

Les conflits autour d'œuvres littéraires survenus au cours de la première moitié de ce siècle et en particulier dans les années vingt et trente sont passés en revue par Ragâ' alNaqqâch, directeur d'al-Musawwar, comme autant de témoignages de la tolérance qui existait selon lui à l'époque considérée $e^{79}$. Il n'est pas sans intérêt de considérer l'échange polémique auquel cet article donne lieu dans un numéro suivant de la revue, avec 'Isâm al-Aryàn, secrétaire général adjoint du syndical des médecins et membre de la direction de l'Association des Frères musulmans ${ }^{80}$, dans la mesure où l'invocation des années trente ne peut se faire qu'au prix d'une relecture partielle et partiale, où les arguments sont facilement réversibles, compte tenu des enjeux qu'ils prétendent servir dans l'Égypte actuelle. Partant de ces exemples, Naqqâch estime que du point de vue de 
la liberté de publier, la période actuelle est une « régression » et que, de plus, la censure est "vaine ». Pour Aryân, c'est cette liberté des années vingt et trente qui démontre l'absence de liberté actuelle. Argument irrecevable pour le rédacteur d'al-Musawwar qui, pris au piège de sa relecture, doit en infirmer les conclusions précédentes et vanter les mérites de la démocratie moubarakienne.

Quoi qu'il en soit, l'analyse faite par Naqqâch de chacun des exemples proposés le conduit à affirmer, d'une part, qu'à l'époque considérée ces conflits se sont résolus par le dialogue beaucoup plus que devant la justice, et d'autre part, que les auteurs qui ont ainsi été convaincus d'excès et d'erreur se sont « volontairement » amendés : la liberté de création est absolue en principe, mais à condition qu'elle ne menace pas un consensus sur les règles, sans lequel l'unité nationale est menacée. Quels sont ces écrivains ? Taha Husayn d'abord : son ouvrage contesté sur la poésie anté-islarmique (1926) a été soumis à une enquête judiciaire qui a abouti à un non-lieu, le procureur Muhammad Nûr ayant estimé que «la sanction de l'erreur relève du tribunal de l'opinion». Le livre fut en fait racheté en bloc par l'Université dont Lutfi al-Sayyid était le président, et Taha Husayn publia ultérieurement une nouvelle édition, amendée, sur la «littérature » antéislamique. Bien qu'anti-wafdiste, note Naqqâch, T. Husayn fut soutenu notamment par Abbas al-'Aqqâd, proche du Wafd. Mansur Fahmi ensuite, auteur d'une thèse iconoclaste soutenue en France (1913) sur «La femme dans l'islam »". Ce livre « faible », "dirigé contre l'islam et ses prophètes", "rédigé en français sans traduction arabe, sous la direction d'un professeur juif, Henri Lévy-Bruhl, adversaire forcené de l'islam et des musulmans ${ }^{8_{2}}$, fit l'objet d'une réponse de Muhammad Lutfi Gum'a, qui traduisit en arabe et critiqua son livre :

«Mansur Fahmi ne put rien faire devant les réfutations de M. L Gum'a. Il se réfugia dans le silence et même se retira de la vie publique pendant un certain temps pour s'occuper d'agriculture. Après quoi il se remit à écrire et à enseigner à l'Université, devint directeur de Dâr at-kutûb (Bibliothèque nationale) et occupa de nombreuses autres fonctions. Mais il avait alors modifié sa pensée et compris la réalité de sa faute. Dans ses nouveaux écrits, il était devenu un défenseur jaloux de l'islam. Et tout cela sans qu'il ait été exposé à aucune poursuite judiciaire. ${ }^{83}$ "

Tragique en revanche fut le sort d'Isma'îl Adham, auteur d'un ouvrage intitulé « Pourquoi je suis athée ». Le livre trouva un imprimeur et on pouvait se le procurer sur le marché ${ }^{84}$ :

«La société égyptienne n'en fut pas ébranlée et l'État n'engagea pas de poursuites judiciaires; mais il y eut un écrivain islamique pour répondre à Adham par un livre intitulé Pourquoi je suis croyant. C'était une réponse objective, en termes mesurés et précis, qui «annula» les effets du livre d'Adham. Bien plus, l'affaire se solda, quelques années plus tard, par le suicide d'Adham. »

Là encore, il n'y eut pas de procès. De même, Chibli Chumayyil (1853-1917) avait pu diffuser ses idées sur le darwinisme sans être inquiété. En dépit de «leur absurdité et de leur fausseté ", ses théories furent discutées par les oulémas. Le cheikh Tantawi alJawhari et Muhammad 'Abduh, sans déclarer ces théories antireligieuses, "l'emportèrent sur Chumayyil' en prouvant "rationnellement" leur absence de fondement intellectuel. Au lendemain de la deuxième guerre mondiale, d'autres débats eurent lieu; en 1945, l'ouvrage de 'Abd al-Rahmâm Badawi sur L'histoire de l'athéisme en islam fut également discuté sans être saisi comme ce fut le cas, en 1947, de la thèse de Muhammad Ahmad Khalaf Allah sur L'art narratif dans le Coran, qui reçut une réponse de la part du cheikh d'al-Azhar, Mahmûd Chaltût. 

des commentateurs. Ceux, habituellement plus controversés, que cite Naqqâch sont également cités par al-Ahâlit ${ }^{\text {ss }}$ qui y ajoute celui d'Isma'îl Mazhar pour les années trente. Au-delà de cette énumération, c'est en fait l'évaluation des années du mouvement national qui est en jeu. Mais cette évaluation positive de la part d'af-Musawwar, proche du gouvernement, vise un objectif : démontrer que le libéralisme moubarakien pourrait désormais se passer d'un contrôle strict sur la production intellectuelle et se conformer aux exigences démocratiques sur lesquelles il ne cesse de mettre l'accent, et ce parce qu'il est en mesure de s'opposer, avec l'aide d'al-Azhar, à ce que des opinions trop ouvertement "hostiles à l'islam» - c'est-à-dire à l'islam politique - puissent s'exprimer. Tout en compensant ce "libéralisme orienté » par l'autorisation, voire l'encouragement, donnés à des auteurs combattant l'islam politique sans s'attaquer au dogme lui-même (par exemple al-'Achmâwi). La présentation que fait Naqqâsch des années du libéral age est en effet marquée au sceau d'une réislamisation assumée du discours politique. Les seules limitations imposées aux écrivains sont de ne rien publier qui puisse troubler la conscience des musulmans égyptiens. Si cela était, le « vrai » peut toujours être distingué du faux par le "dialogue", les oulémas sont là pour en administrer la « preuve » et les écrivains pour reconnaitre leur « erreur ».

Ce scénario pourtant pose problème, comme le prouve la réaction de 'Isâm al-'Aryân dans une lettre adressée à Naqqâch. "A qui s'adresse ce discours contre la censure? A l'État, dont al-Azhar est une institution très dépendante? Aux intellectuels en général ou à ceux qui défendent l'islam et sont, de ce fait, persécutés?" interroge le dirigeant des Frères musulmans avant d'ajouter ${ }^{86}$ :

«Où peut-on trouver ce climat de dialogue dans la nation? Des essais et des œuvres littéraires pouvaient être produits grâce au climat de liberté dont les Egyptiens jouissaient - Il faut malheureusement le dire - sous la domination de l'Angleterre et d'une monarchie corrompue. Que reste-t-il aujourd'hui d'un tel climat ? Où est la liberté d'éditer des journaux et des revues? N'avez-vous pas entendu parler, Monsieur, de ces journaux qu'on importe et qui sont rédigés en Égypte, mais dont l'autorisation est délivrée à Chypre ou ailleurs ? Pourquoi tous ces détours et ces cheminements tortueux?»

La question, on le voit, porte moins sur les années trente que sur les années Moubarak, Elle est du reste prolongée par un rappel de l'interdiction des partis politiques et de l'absence de réelle démocratie. Mais l'évaluation de la première période et surtout de la liberté supérieure dont auraient joui les Egyptiens, malgré leur assujettissement, est lourde de sens. Et la réponse est immédiate, laissant apparaître sous la plume de Naqqâch qu'en effet, les choses ne sont pas aussi simples. Non sans avoir auparavant interpellé 'Aryân sur son "amnésie » et sur son "étrange conception de l'histoire », il présente l'envers du décor : l'exil de 'Abduh, de Muhammad Farid, d'al-Gayali ou de Bayram al-Tunsi, l'emprisonnement de 'Aqqâd, la révocation de Taha Husayn de sa charge de doyen, les difficultés faites à Salama Musa ; l'emprisonnement, après 1946, de dizaines de communistes.

Il est donc difficile d'éviter le parallèle avec l'Égypte actuelle et c'est pourquoi l'hebdomadaire al-Musawwar insiste sur une nécessaire compréhension des moments qui jalonnent ce processus de l'accès à une libération de la création intellectuelle. Depuis la révolution nassérienne, l'Égypte n'est ni l'envers, ni l'héritière directe de ces années " éclairées ». Elle s'inscrit seulement dans ce processus continu. Ses institutions ne sont pas «importées ${ }^{87}$ et le moins que l'on doive admettre est qu'elle dispose

Égypte/Monde arabe, 14 | 1993 
désormais d'un État national affranchi de la dépendance externe et garantissant des expressions variées sans mettre en danger l'unité nationale. En affirmant cela, la revue rejoint un certain consensus sur l'unité nationale dont des exemples ont déjà été abondamment cités.

Farag Fawda et l'inutilité de la censure

La question qui était posée sur la vanité d'une censure à laquelle échapperaient beaucoup d'auteurs à condition qu'ils acceptent de se faire publier à Chypre, au Liban ou ailleurs, a été développée par Farag Fawda ${ }^{88}$. Question d'autant plus cruciale que Fawda publiait régulièrement en Égypte, à l'instar de quelques autres auteurs, des analyses contestées par beaucoup de ses confrères, qui remettaient en cause le consensus auquel se sont bon gré mal gré ralliés un certain nombre d'intellectuels, compte tenu notamment de la présence croissante sur la scène politique des mouvements de l'islam politique. L'hebdomadaire al-Nûr rappelle par exemple l'hostilité ouvertement manifestée par Fawda aux programmes de réislamisation des institutions ( $\mathrm{Je}$ suis opposé a l'application de la charîa islamique, que ce soit immédiatement ou par étapes ») et s'indigne, nous l'avons vu, de la réédition des œuvres complètes de l'écrivain après sa mort en refusant d'en faire un "martyr » de la liberté $\mathrm{d}^{\prime}$ expression ${ }^{89}$; tandis que d'autres s'étaient opposés à sa critique du nassérisme et de ses avatars, faite au nom des acquis du wafdisme, ou à son approbation de la politique d'ouverture initiée par Sadate à l'égard d'Israël.

C'est en particulier sur Salman Rushdie que Fawda a explicité ses vues, s'étonnant de l'amnésie de beaucoup quant à l'histoire islamique: "L'époque abbasside a connu des dizaines d'athées à côté desquels Salman Rushdie n'est que menu fretin". " Le mu'tazilite Ibn al-Rawândi (mort en 864) a écrit bien pire sur le Coran et la sunna, et on pourrait en dire autant d'al-Tawhidi et d'Abu-1-'Ala al-Ma'âri. Si leurs écrits cependant ne sont pas restés, en dehors de la connaissance qu'en ont quelques spécialistes, il n'en est pas de même pour al-Hallaj, martyr, toujours présent dans la mémoire du soufisme: l'interdiction et sa mort ont échoué à censurer sa pensée ${ }^{91}$. On peut en dire autant des hommes de science européens dont les idées se sont en fin de compte imposées " par la libre discussion »: Galilée, Copernic, Darwin ou Freud'2. En Égypte, de même, 'Ali 'Abd al-Râziq est toujours discuté et le Parti Wafd, dont l'histoire fut occultée plusieurs décennies durant, est revenu sur la scène politique.

Un des paradoxes de cette situation, selon Fawda, est que les auteurs censurés et dont les œuvres sont "exilées» ont vu décupler leurs ventes, comme dans le cas de Mahfouz, «alors que ses concitoyens ne peuvent lire les plus importants de ses livres, qui ont été récompensés du prix Nobel ${ }^{\text {s" }}$ ». La censure ne parvient donc pas à détruire les constructions intellectuelles qui s'imposent dans le temps, mais les phénomènes d'amnésie collective, fussent-ils temporaires, interrogent les intellectuels sur leur propre responsabilité. Sur ce point, Fawda est rejoint par différents auteurs dont nous allons, pour conclure ce « dossier », examiner le sens de la critique.

Les clercs ont-ils trahi?

62 La question vient du philosophe Murad Wahba. Pour y répondre, il se place sur le terrain de l'étude du turâth et des utilisations qu'en font les hommes de lettres. Son interprétation de l'héritage s'inscrit, dit-il, dans une histoire dont la Grèce est le point de départ. La raison humaine est qualifiée pour interpréter tout texte religieux, en dehors de la pression de l'autorité religieuse. Il y a eu, dit-il, dans la Nation arabe, des 
intellectuels éclairés - Taha Husayn, 'Ali 'Abd al-Râziq, Naguib Mahfouz et autres Louis 'Awad - mais la censure de leurs livres a interdit qu'un « courant des Lumières » puisse exister $^{94}$. C'est donc à l'intérieur qu'il faut se placer pour mesurer le problème ${ }^{95}$ :

«L'un des dommages causés par le fait de mêler le colonialisme et la culture est que les mouvements de libération nationale ne se sont pas occupés de réfléchir sur les germes du retard qui les touchait en propre, mais seulement sur les facteurs externes de ce drame. La preuve en est que l'on se demande aujourd'hui où sont passés ces mouvements. "

Doit-on pour autant parier de "trahison des clercs »? Se méfiant de la formule, Murad Wahba considère néanmoins que l'intellectuel se contente d'exercer son esprit critique sur la politique au quotidien, alors que « le problème fondamental n'est pas dans le conflit de l'intellectuel avec le pouvoir, mais avec une société attardée. Sa fonction est de découvrir les racines de ce retard qui ne sont pas à l'extérieur, mais à l'intérieur ». En s'abstenant d'agir, il s'est mis en marge de la sociétés.

Une marginalisation qui expliquerait une propension à la division au sein de l'intelligentsia ? Faisant le bilan de l'année 1992, le journal al-Ahâli considère que dans le cas de 'Ala Hamed, où était soulevée la question de la liberté d'expression, « la presse a joué à la fois le rôle du procureur et de l'avocat" ". Si l'on excepte les oulémas et les Frères musulmans, qui affirment que la censure n'est pas bonne dans son principe, mais est nécessaire et doit être régie par une loi claire, la plupart des intellectuels se sont rangés aux côtés des écrivains menacés ou persécutés. Ce qui ne rend que plus visibles ceux qui se sont ouvertement prononcés contre tel ou tel écrivain : nous l'avons vu avec Ahmad Bahgat, chroniqueur d'al-Ahrâm, mais aussi avec Tharwat Abaza, président de l'Union des écrivains dont certains des membres cotisants de l'Union ont ressenti comme une attaque les explications qu'il a demandées, après une délibération du bureau de l'Union, au président de la GEBO, sur les raisons pour lesquelles ce dernier avait autorisé la publication, dans une des revues qu'elle édite (Ibdâ'), du poème controversé de Hassan Teleb. Preuve supplémentaire, pour ces critiques, d'une division inquiétante des écrivains, l'Assemblée de l'Union avait, semble-t-il, refusé que le texte contesté soit soumis à al-Azhar, mais accepté le principe de l'intervention auprès de la $\mathrm{GEBO}^{98}$.

C'est néanmoins sur le terrain de la confrontation avec le pouvoir politique que peut avoir lieu, pensent certains, la réconciliation des intellectuels avec leur société, pour nuancer les propos de Murad Wahba. Faute de pouvoir fonder une légitimité univoque de l'acte d'écrire et d'émettre son opinion, cette exigence impose à l'intellectuel de se battre pour une publicité des débats et pour une transparence des procédures sans laquelle aucune définition d'une authentique légalité du contrôle des écrits ne peut être mise en œuvre, L'écrit seul doit répondre à l'écrit et l'autorité politique ne doit intervenir que comme arbitre et non comme instance de répression au gré des pressions ou du « lobbying » politique ou religieux. Beaucoup des auteurs cités pensent qu'il est essentiel de "maintenir le lien social», mais croient aussi que l'histoire égyptienne doit faire l'objet d'une relecture plurielle et contradictoire. Comme le dit Murad Wahba, c'est en soumettant à la critique le concept de nahda que le pacte social devrait être repensé ${ }^{\prime 90}$ :

«La nahda signifie renaître, c'est-à-dire faire une relecture du turâth qui donne la capacité aux valeurs positives de représenter l'avenir. La vision prospective de la nahda ne s'incarnera ni dans les Lumières ni dans le laïcisme. Je demande donc à 
ceux qui utilisent ces concepts ce qu'ils entendent par là. Je crains en effet que nous ne réinvestissions des concepts occidentaux sans en connaître l'histoire et la façon dont ils ont été employés, à la manière des erreurs qui ont accompagné l'emploi du concept de Lumières. Le renouvellement de ces concepts revient aux philosophes, mais s'ils démissionnent, comment sortirons-nous de l'impasse? »

\section{NOTES}

1. Oktober, $27 / 3 / 92$.

2. C'est le cas, par exemple, du premier ouvrage de Muhammad Saïd al-'Achmawi, Usûl al-charîa, publié en 1979, saisi une première fois en 1983, mis de nouveau sur le marché, attaqué en 1987 par al-Azhar, interdit une seconde fois puis de nouveau en vente.

3. Comme c'est le cas pour Naguib Mahfouz.

4. Cf. Hamdi Surur, in Rose af-Yussef, 11/1/93.

5. Mustala Darwich, « La censure, le cinéma et d'autres choses », Bulletin du CEDEJ,

21,1987.pp, 97-107.

6. Cité par al-Ahâli, 30/12/92.

7. Ibidem.

8. Akhbâr al-Yawm. 20/6/92.

9. Al-Ahrâm, 3/1/92.

10. Al-Ahrâm, 6/1/93.

11. Al-Ahrâm al-duwali. 17/1/92.

12. Al-liwâ' al-islâmi, 9/1/92.

13. Cf. la relation de cette rencontre du $9 / 1 / 92$ dans Adab wa naqd n $78,1992$.

14. Al-Siyâsi, 21/1/92.

15. Al-Abrâm, 13/1/92.

16. Lbid.

17. Un ancien président de l'Académie des recherches d'al-Azhar, 'Abd al-Galîl Chalabi, rappelle que « certains ouvrages que l'Académie a recommandé de saisir portent .atteinte au droit de la religion islamique en la mettant sur un pied d'égalité avec de nombreuses autres religions comme le bouddhisme ou le confucianisme. » Al-Cha'b, 28/1/92.

18. Comme il le dit dans une interview donnée à al-Ahâli, 15/1/92.

19. Al-Ahâli, 5/2/92, 'Alim islâmi sâdarahu al-Azhar (Un savant en sciences islamiques interdit par al-Azhar).

20. Voir, parmi d'autres, le point de vue de Raga' al-Naqqach dans al-Musawwar des $21 / 2 / 92$ et $27 / 3 / 92$.

21. Al-Ahrâm al-duwali, 17/1/92, art. cit.

22. Al-Cha'b, 28/1/92.

23. Dâr al-ma'ârif a également réédité plusieurs livres de l'écrivain.

24. Hassan Teleb est l'auteur d'un recueil de poèmes qui fut retiré de la vente après que l'Académie des recherches islamiques de l'Azhar eut porté sur lui l'appréciation suivante : «De nombreux vers du recueil présentent des similitudes avec des versets du Coran. » Rose al-Yussef, 11/1/93. 
25. Rose al-Yussef, 11/1/93.

26. Al-Nûr, 19/8/92.

27. Faruq Hosni, in al-Ahâli, 8/1/92.

28. Pour reprendre la dénonciation de Tharwat Abaza, cf. supra.

29. En dehors de quelques réactions remarquables comme celle de Muhammad Khalaf Allah ; cf. le dossier sur cette affaire dans la Revue de la presse égyptienne $\mathrm{n}^{\circ} 36-37,1989$.

30. Al-Cha'b, 28/1/92.

31. Voir les points de vue sans équivoque, dans les grands quotidiens, de Ghali Chukri, Lutfi al-Khuli, Sami Khachaba, Yusuf al-Qa'id, Ra'fat al-Sa'îd, pour citer les principaux (période du 1er au 9/1/92).

32. Cf. al-Ahrâm du 23 mars 1989, "Le pluralisme, non la transgression ».

33. Al-Cha'b, ibid.

34. Ibid

35. Ibid

36. Al-Cba'b, 4/2/92

37. In Rose al-Yussef. 2/1/92.

38. Cf. l'interview de 'Achmâwi dans al-Ahâli, 15/1/92, approuvée par Adab wa naqd, $\mathrm{n}^{\circ} 78,1992$.

39. Ce type de défense est courant dans plusieurs périodiques déjà cités, notamment dans Rose al-Yussef, al-Ahâli el Adab wa naqd.

40. Dont le livre MakhIûqâa a-achwâq al-tâ'ira a été retiré de la vente par la GEBO, son propre éditeur.

41. Auteur de Al-'Urât, également interdit à la Foire du livre de 1992.

42. Rose al-Yussef, 6/1/92.

43. Al-Ahrâm. 8/12/91.

44. Selon Adab wa naqd, 78/1992.

45. Al-Ahrâm al-duwali, 3/3/90.

46. Rose al-Yussef, 2/1/92.

47. Ibid

48. Al-islâm al-siyâsi [L'islam politique], Le Caire, Sîna li-l-nachr, 1987.

49. T. al-Châwi, Qadiyati ma'a kutub al-'Achmâwi [Mon différend avec les livres de 'Achmâwi], al-Cha'b, 4/2/92; cf. également, « C'est moi qui ai dénoncé le conseiller 'Achmâwi à al-Azhar ", Akhbâr al-Yawm, 1/2/92.

50. Al-Ahâli, 5/2/92.

51. Notamment, les prophètes dans le Coran, Le musulman obéissant ; est-ce qu'il sort de la lumière pour entrer dans le feu de l'enfer? et L'Égypte dans le Coran, ibid.

52. Ibid.

53. Ibid.

54. Constitution de 1971, modifiée en 1980, Titre II ; Les libertés, les droits et les devoirs publics, articles 47 et 48 .

55. Cf. Akhir Sâ'a, 22/1/92, le point de cette question par M. S. al-'Achmâwi.

56. Art. 15 de la loi ${ }^{\circ} 103$ de 1961.

57. Al-Akhbâr, 14/2/92.

58. Point de vue exprimé par l'Organisation égyptienne des droits de l'homme (OEDH), al-Akhbâr. 22/1/92.

59. Cité par Adab wa naqd, ibid.

60. Ibid

61. Al-Ahâli, 30/12/92. 
62. Rose al-Yussef, 11/1/93

63. lbid.

64. Al-Cha'b, 4/2/92.

65. Al-Ahâli, 5/1/92.

66. Al-Ahrâm, 20/6/92.

67. Al-Siyâsi, 12/1/92.

68. Al-Ahrâm al-duwali, 17/1/92.

69. Fâruq Husni, qui fait observer dans le même mouvement le progrès réalisé par rapport à l'époque nassérienne, al-Siyâsi, ibid.

70. Fahmi Huwaydi, cité par Rose al-Yussef, 11/1/93.

71. $C f$. l'interview précédemment citée.

72. Adab wa naqd, ibid., p. 26.

73. Al-Ahâli. 6/1/93.

74. Al-Akhbâr, 22/1/92.

75. Cité par Adab wa naqd, ibid.

76. Alors que le cheikh d'al-Azhar condamne en termes très généraux, au lendemain de la mort de Fawda, le recours à la violence et à l'assassinat politique, 'Abd al-Ghaffâr 'Aziz, ancien doyen de la Faculté de da'wa d'al-Azhar, s'en prend violemment, dans une déclaration et un article, à l'écrivain. Avant l'assassinat, Muhammad al-Birri el Tal'al al-Birri, enseignants de cette Faculté, avaient dénoncé l'écrivain et le « danger pour l'islam » que représentait le Hizb al-mustaqbal, le parti dont il demandait la reconnaissance par le gouvernement ; $c f$. François Zabbal, «L'assassinat de Farag Fawda ", les Cahiers de l'Orient, n²7, 1992, pp. 169-198.

77. Rose al-Yussef, 2/1/92.

78. Al-Ahâli, 8/1/92.

79. Al-Musawwar, 21/2/92.

80. Al-Musawwar, 27/3/92.

81. Rééditée sous le titre La condition de la femme en Islam, Paris, 1991.

82. Al-Musawwar, 21-2/92.

83. Ibid.

84. Ibid.

85. Al-Ahâli, op. cit.

86. Lettre publiée par al-Musawwar du 27/3/92.

87. Ibid.

88. Oktober, $27 / 3 / 92$.

89. Al-Nûr. 19/8/92.

90. Al-Ahrâm. 26/8/91.

91. Oktober, ibid.

92. Ibid.

93. Déclaration de l'OEDH, Al-Akhbâr, 22/1/92.

94. Al-Ahâli, 5/2/92.

95. Ibid.

96. Ibid.

97. Al-Ahâli, 30/12/92.

98. Rose al-Yussuf, 18/1/93.

99. Ibid. 
INDEX

Mots-clés : al-Azhar, censure, édition, islam, littérature, ouléma, religion, Nahda

\section{AUTEUR}

BERNARD BOTIVEAU

IREMAM, Aix-en-Provence 